\title{
Operational Stability and Degradation of Organic Solar Cells
}

\author{
Efe Çetin YILMAZ1 ${ }^{1}$, Muhammet Kaan YEŞILLYURT², İlhan Volkan ÖNER², Gökhan ÖMEROĞLU \\ Ahmet Numan ÖZAKIN ${ }^{3}$ \\ ${ }^{1}$ Ataturk University Pasinler Vocational School, Department of Electronics and Automation \\ ${ }^{2}$ Ataturk University Pasinler Vocational School, Department of Electricity and Energy \\ ${ }^{3}$ Ataturk University Engineering Faculty, Department of Mechanical Engineering
}

\section{Article Info \\ Article history: \\ Received May $25^{\text {th }}, 2017$ \\ Revised June $14^{\text {th }}, 2017$ \\ Accepted June 16 ${ }^{\text {th }}, 207$}

\section{Keyword:}

Degradation

Efficiency of organic solar

cells,

Stability,

Life-cycle of OSCs

\begin{abstract}
Recently, Organic solar cells (OSC) have been increasingly utilized all over the world. The changes made in the organic components of the organic solar cells enable them to exhibit good features such as mechanical flexibility, lightness and high power generation efficiency even under lower light intensities. However, operational stability is an important parameter for organic solar cells. Despite the aforementioned advantages of organic solar cells, degradation in operational environments limits their use in harsh conditions. Studies have shown that the organic layer and the cathode layer of the OSCs are degraded by external factors, and this adversely affects the operational stability and productivity of OSCs considerably. The overall efficiency of an organic solar cell is defined as a function of life cycle and efficiency of energy generation. Therefore, the shorter the life cycle becomes, the lesser the overall efficiency of OSCs gets. Recent studies are focused on improving the operational stability and power generation efficiencies of OSCs by reducing the effects degradation induced by external factors, such as climatic conditions and thermal fatigue. The purpose of this study is to assess how organic solar cells work, how they degrade from external factors, such as water and water vapor, and how these parameters affect the operational stability as well as the efficiency of the organic solar cells.
\end{abstract}

\section{Corresponding Author:}

İlhan Volkan ÖNER,

Pasinler Vocational School, Department of Electricity and Energy Ataturk University 25200 Erzurum, Turkey

Email: ivoner@atauni.edu.tr

\section{Introduction}

This study is part of our researches and studies on renewable energy systems in identifying the problems in their utilization and seeking solutions to them. In this study we tackle the stability and degradation mechanisms of organic solar cells. Organic solar cells are very promising for their superior characteristics such as mechanical flexibility, lightness and high power generation efficiency even under lower light intensities.

Recently, Organic solar cells (OSC) have been increasingly utilized all over the world. The changes made in the organic components of the organic solar cells enable them to exhibit good features such as mechanical flexibility, lightness and high power generation efficiency even under lower light intensities. However, operational stability is an important parameter for organic solar cells. Despite the aforementioned advantages of organic solar cells, degradation in operational environments limits their use in harsh conditions. Studies have shown that the organic layer and the cathode layer of the OSCs are degraded by external factors, and this adversely affects the operational stability and productivity of OSCs considerably [1]. 
The overall efficiency of an organic solar cell is defined as a function of life cycle and efficiency of energy generation, which is mostly referred to as Power Conversion Efficiency (PCE). Therefore, the shorter the life cycle becomes, the lesser the overall efficiency of OSCs gets. Recent studies are focused on improving the operational stability and power generation efficiencies of OSCs by reducing the effects of degradation induced by external factors, such as climatic conditions and thermal fatigue [1].

The main objective in solar cell researches is, for sure, to bring in solar systems having high power conversion efficiency. With the introduction of fullerene electron acceptors and other new approaches, organic solar cells have improved efficiencies remarkably in the last decade $[1,2]$. On the other hand, the absorption band of OSC within the solar spectrum is now broader than ever [3]. The total yield from a solar cell is calculated by its efficiency multiplied by its lifetime. Therefore, not alone he high efficiency, but also the longer lifetime is important. And no doubt, in order for the lifetime to be longer, stability and degradation resistivity are important parameters that also affect their value (yield over cost) as current energy production is largely limited by the low durability.[1] In the recent years, by means of the changes made in the internal structure of the solar cells and thanks to the newly introduced synthetic materials, noteworthy improvements have been achieved in terms of operational stability of organic solar cells [1].

In recent years, numerous internal structures have been developed that can be less affected by degradation factors. In addition, improvements have been made to the operating stability and lifetime of OSCs operating under constant light intensity [4]. In modern OSCs manufactured with an aluminum-titanium top cathode layer, operational stability and efficiency is improved [1]. With this improvement, degradation rate of OSCs in air environment has been slowed down.

The purpose of this study is to assess how organic solar cells work, how they degrade from external factors, such as water and water vapor, and how these parameters affect the operational stability as well as the efficiency of the organic solar cells. State the objectives of the work and provide an adequate background, avoiding a detailed literature survey or a summary of the results.

\section{Material and methods (Working Principle of Organic Solar Cells)}

Organic solar cells (OSCs) are considered to be a green solar energy technology. OSCs are attractive mainly because they can be fabricated from various organic compounds with modifiable structures and exhibit excellent flexibility, high power conversion efficiency (PCE) under low light irradiation, and promising see-through power-generation windows. Over the course of the past few years, various aspects of organic solar cells have been extensively studied, including synthesis and application of new materials, modeling of physical processes, large-scale manufacturing, stability improvement, et cetera [5].

A single-junction OSC comprises five or six layers stacked on the surface of a supporting substrate, which is normally a piece of glass or transparent plastic. These five or six layers are as illustrated in Fig. 1.

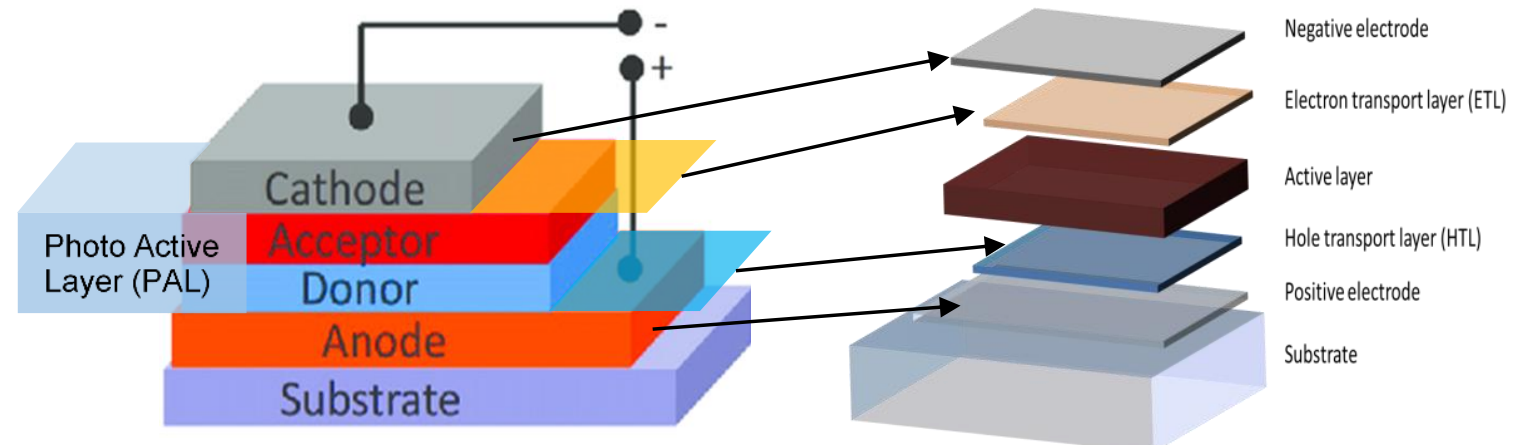

Figure 1. Comparative of a single-junction OSC comprises five or six layers

An OSC consists of:

- A transparent conductive electrode (Cathode, normally of indium-tin-oxide, mostly referred to as ITO),

- Cathode buffer layer (CBL) (also known as electron-transport layer (ETL)),

- one or two photoactive layers (PALs) composed of an electron donor and an electron acceptor,

- Anode buffer layer (ABL) (also known as hole-transport layer (HTL)),

- a top metal electrode (Anode, generally aluminum or silver),

- and a supporting substrate (normally of glass or transparent plastic) 
The HTL also functions as an electron-blocking layer, or an exciton-blocking layer. Similarly, the ETL may also function as a hole-blocking layer. For simplicity, buffer layers are denoted as HTL and ETL in figures throughout the manuscript. An extra encapsulation film is sometimes employed. The structure of these layers are shown in Fig. 1 [1].

In Fig. 2 the electricity generation, namely the working principle of an organic solar cell is illustrated.
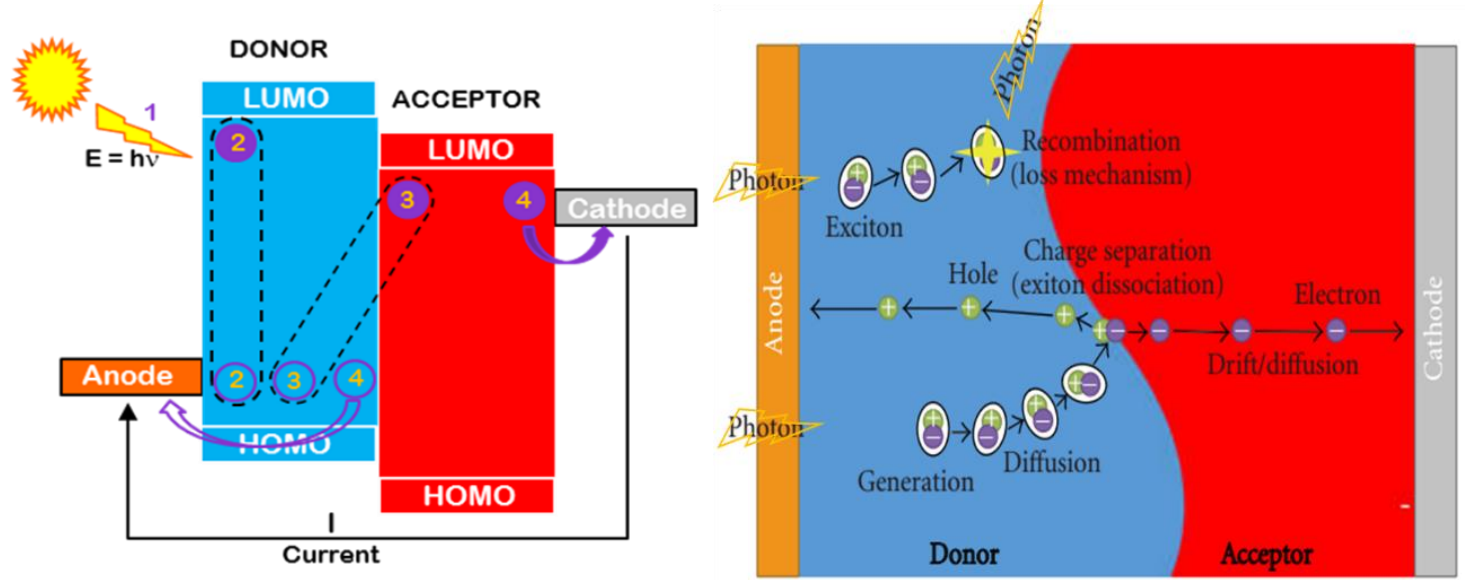

Figure 2. Photocurrent generation mechanism in active layers of an organic solar cell [6]

How photocurrent is being generated in PAL of an OSC:

1) The incident light reaches to OSC

2) An electron is excited by the photon energy (E=hv), and it moves to the higher energy level (LUMO), forming a combination of an electron and an associated hole, an exciton.

3) When an exciton does not reach an interface, it relaxes back into the ground state (loss mechanism) and no current is generated. Therefore, by diffusing to the donor-acceptor interface, the exciton transfers the charge at the interface to the electron acceptor, after which a free electron in the Acceptor and a free hole in the Donor is formed. This mechanism is known as the exciton dissociation.

4) separated charges diffuse to the electrodes, namely the electron reaches the cathode while the hole reaches the anode.

As a result of these four steps, an electric current flows in the opposite direction of the electron flow, namely from cathode to anode. So following challenges should be overcome in order for OSCs to be able to generate photocurrent $[1,7]$ :

-Photons, which generates tightly-bound excitons instead of free charge pairs, should be absorbed

-Since the diffusion lengths of excitons in organic semiconductors are low, donor-acceptor interface should be advanced and charge transfer be made;

-Recombination of excited charges should be avoided at or before the donor-acceptor interface;

-The cooling of hot charge-transfer excitons at the donor-acceptor interface should be prevented

-The low or imbalanced carrier mobilities in semiconductors should be overcome.

Classification of OSCs are generally made by whether the electron donor (D) and acceptor (A) layers are planar or intermixed, and are named as planar-heterojunction (PHJ) or bulk-heterojunction (BHJ), respectively. Illustrated in Fig. 3 is schematic views of these two different types of organic solar cells. The left hand side is the planar heterojunction organic solar cell, and the right hand side is the bulk heterojunction organic solar cells.

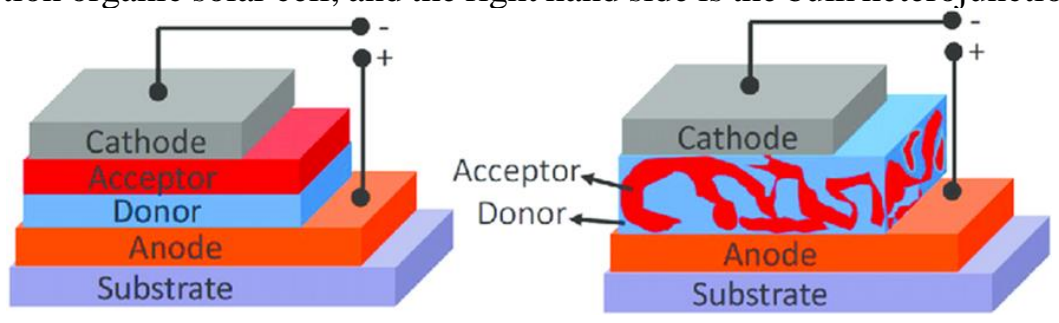

Figure 3. Planar and bulk heterojunction organic solar cells

As you can see here, the acceptor and donor in the active layer are blended and dispersed one in another in bulk systems. So that they can be made thick enough for effective photon absorption without the difficult processing 
involved in orienting a layered structure while retaining similar level of performances. For the PHJ-OSCs, multiple layers of semiconductors are deposited in sequence.

Looking at the application areas of solar cells, a single diode circuit design with two resistances is defined and Shockley equation is obtained. This circuit is described below, in Fig. 4.
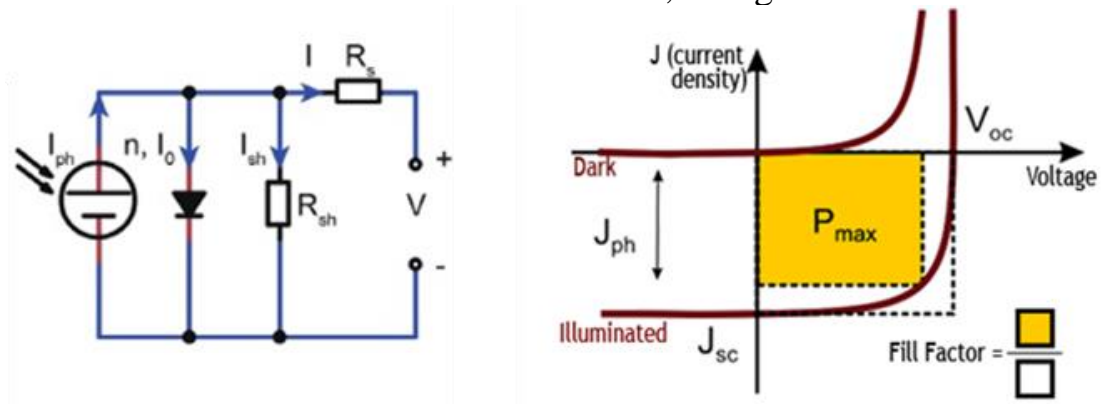

Figure 4. (a: [1]) Single diode solar cell under irradiance (b: [8] I-V curve of a solar cell in dark and under sunlight

$$
I-I_{0} \times\left[e^{e \times\left(\frac{V-I \times R_{S}}{n k_{B} T}\right)}-1\right]-\frac{V-I \times R_{S}}{R_{p}}+I_{\text {light }}=0
$$

It gives the I-V (current-voltage) characteristic of an idealized diode in either forward or reverse bias (applied voltage) In this equation, I is the diode current, $\mathrm{V}$ is the externally applied voltage, $\mathrm{I} 0$ is the current at saturation point, $T$ is the temperature, $R_{s}$ is the serial resistance, $R_{p}$ is the parallel resistance, $k_{B}$ is the Boltzmann constant and I light is the light induced electrical current. The $R_{s}$ in the series refers to the resistance of serial resistance semiconductors and the resistance to noise resistance of the connection electrodes. The $R_{\mathrm{s}}$ value should ideally be zero. If the $R_{s}$ resistance is too high or $R_{s h}$ has very low values, the solar cell will be damaged. Therefore, these parameters should be calculated using Eq. (1) and from the single diode I-V curve given in Fig. 4 [1]. Moreover, the actual I-V curve for OSCs differentiates from the ideal I-V curve [9] Qi and Wang summarized factors influencing the open circuit voltage $\left(\mathrm{V}_{\mathrm{oc}}\right)$ and fill factor $(\mathrm{FF})[10,11]$. While open circuit voltage is also a function of a number of things such as temperature, irradiance and material microstructure, it is briefly determined by the difference between the energy levels where the exciton dissociation takes place. Figure 4.(b) shows the I-V curve along with FF, $\mathrm{V}_{\text {oc }}$ and short circuit current, theoretical max power indications. FF loss is typically indicated by the appearance of the S-shaped I-eV curve, which is caused by the electric field formed by accumulated charges in devices. Scharber et al. suggested that a donor material with a HOMO of $5.4 \mathrm{eV}$, and a bandgap of $1.4 \mathrm{eV}$ is most suitable for coupling with fullerene to achieve a theoretical PCE of upto $10 \%$ [12].

\section{Degradation of Organic Solar Cells}

Degradation of organic solar cells refers to the deterioration in their operational capacity. This deterioration does not necessarily occur always in the chemical structure of the OSC's. The degradations of OSC's can be subdivided into two groups, degradation under the effects of internal structure and induced by external factors. The significant part of the degradation is caused by the ambient air. Mass transfer (diffusion) is a concern in both degradation types. For example, OSCs having metal-organic intermediates stored under inert atmosphere environment has been shown to suffer significant degradation.

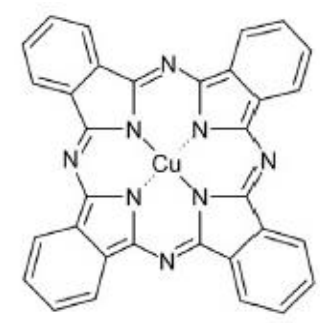

a) $\mathrm{CuPc}, \mathrm{C}_{60}$ and $\mathrm{P} 3 \mathrm{HT}$

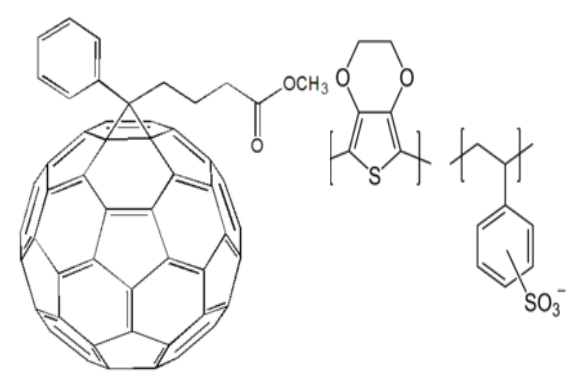

b) $\mathrm{PCBM}^{1}$ and PEDOT:PSS ${ }^{1}$

Figure 5. Chemical structures of OSCs built using common semiconductors (a: [1]; b: [13]) 
In organic solar cells, oxygen and water are two basic external factors that cause degradation. In the literature these two factors are mostly discussed. Oxygen is thought to be a dominant degradation parameter in organic solar cells, which is explained in the following way. Firstly, the water is not easily penetrating to the interior of the OSC. Secondly, due to the structure of the electron transport layer (ETL), the effect of oxygen is more visible and it directly affects the operating efficiency of the OSC. Third, it worsens operating stability of conventional OSCs as a result of damaging metal-formed interface layers [1].

\begin{tabular}{|c|c|c|}
\hline Degradation Mechanisms & Likelihood & Approaches \\
\hline Diffusion of oxygen and water & High & $\begin{array}{l}\text { Device encapsulation } \\
\text { Low OTR/WVTR } \\
\text { adhesives }\end{array}$ \\
\hline Photo-oxidation of polymers & Medium & $\begin{array}{l}\text { Selection of stable active } \\
\text { polymers; Device design }\end{array}$ \\
\hline $\begin{array}{l}\text { Electrode degradation (ITO and } \\
\text { metal) }\end{array}$ & High & $\begin{array}{l}\text { Optimization of metal } \\
\text { electrode }\end{array}$ \\
\hline $\begin{array}{l}\text { Buffer layer degradation } \\
\text { (PEDOT:PSS) }\end{array}$ & Medium & Optimization of $\mathrm{HTL} / \mathrm{ETL}$ \\
\hline $\begin{array}{l}\text { Mechanical degradation } \\
\text { (delamination, etc.) }\end{array}$ & Low & $\begin{array}{l}\text { Adhesion improvement } \\
\text { and lamination }\end{array}$ \\
\hline
\end{tabular}

Figure 6. Degrading mechanisms of OSCs and their likelihood to occur by [14]

Traditional OSCs would highly degrade under atmospheric conditions. Degradation Mechanisms and their likelihood of occurrence is given in the below table, which is taken from Solarmer Energy publications. As you can see, the high likelihood of occurrence is subject for Oxygen and Water along with the electron degradation. Therefore, as we tackle degradation from external factors, the only parameter to consider is water and oxygen. As for some other materials being used in OSCs, oxygen may offer an also constructive effect in terms of electronic properties, since oxygen is typically a p-type dopant in semiconductors $[1,15]$, and oxygen vacancies serve as electron donors [16]. For that reason, for layers wherein the majority carrier are holes, the electronic properties may temporarily be enhanced when exposed to oxygen. Lira-Cantu et al. fabricated inverted organicinorganic hybrid solar cells (HSCs) with a structure of ITO/oxides/MEH-PPV/Ag, and found that applying the semiconductor oxides, such as $\mathrm{ZnO}$, or $\mathrm{TiO}_{2}$, or $\mathrm{Nb}_{2} \mathrm{O}_{5}$, requires the presence of oxygen for the correct functioning of the semiconductor oxides as electron acceptors.[15] These HSCs degrade drastically under light irradiation in vacuum, but are partially recovered upon exposure to air.[1, 17] They proposed that the degradation is due to the removal of oxygen from the $\mathrm{Nb}_{2} \mathrm{O}_{5}$ surface under UV irradiation, whereas the recovery of performance in air comes from the reuptake of oxygen.[18]. Later, they proposed another oxygen-related mechanism to explain the photo-activation of OSCs, in which $\mathrm{ZnO}$ was used as the ETLs. In this case, however, the photoconductivity of $\mathrm{ZnO}$ was proposed to be activated upon exposure to UV irradiation .[19]

Oxygen-induced degradation in Organic solar cells is of external environment based degradations. This parameter is often discussed in the literature. Oxygen is dominant parameter in organic solar cells because of the factors given below:

-While allotrope materials do not react with water, they can easily react with the oxygen in the atmosphere. For example, while aluminum exhibits high strength in aqueous media this behavior is not possible with oxygen in the atmosphere.

-In allotrope materials oxygen can enter the electron transport layer very easily and lowers the operational efficiency of this layer. Improvements made on surface of metallic materials to prevent formation of pitting on these surfaces and hence the damage as a result of reaction with oxygen

In the literature, it has been explained in many studies that this parameter cause degradation to a lesser extent when compared to oxygen. While surface of Aluminum is observed to exhibit a good passivity in dry air, degradation on the surface is observed in a humid environment, however the degradation is shown to be quite small when compared to the degradation rate of oxygen.[1] 


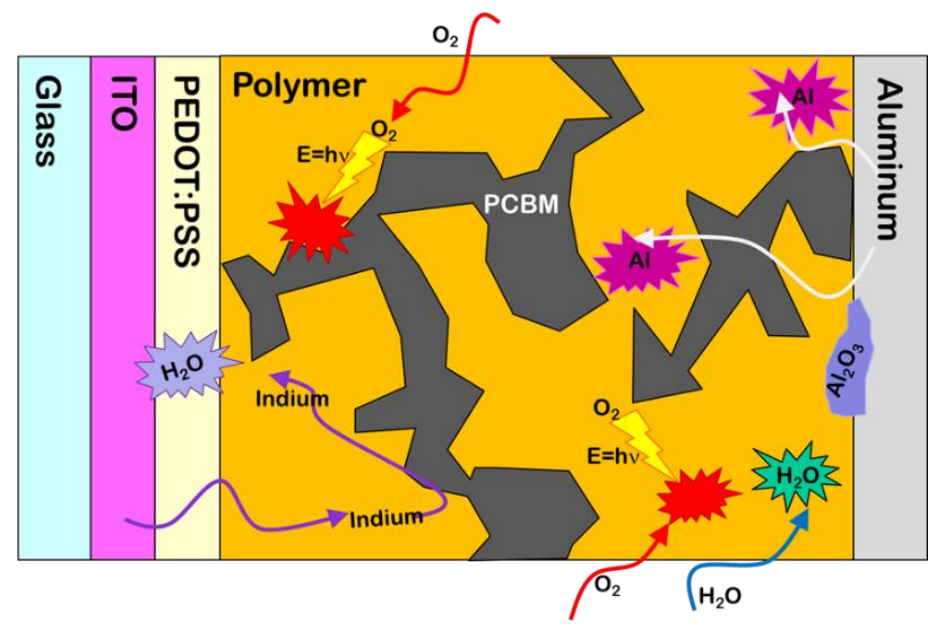

Figure 7. Schematic illustration of degradation with oxygen and water

OSCs manufactured in conventional architecture suffered severe degradation in air environment[20]. Later OSCs were begun to be produced with different techniques in order to reduce their susceptibility to hostile environments. And researches have revealed that a change in the planar and polar arrangement of the organic solar cells significantly reduce their vulnerability in such environments, and inverted OSC architecture was introduced. An Inverted organic solar cell is an OSC with its ETL and HTL is swapped along with the Anode and Cathode. Thus, we can classify the OSCs as conventional (anode directly on substrate) or inverted (cathode directly on substrate) according to which side is close to the substrate.
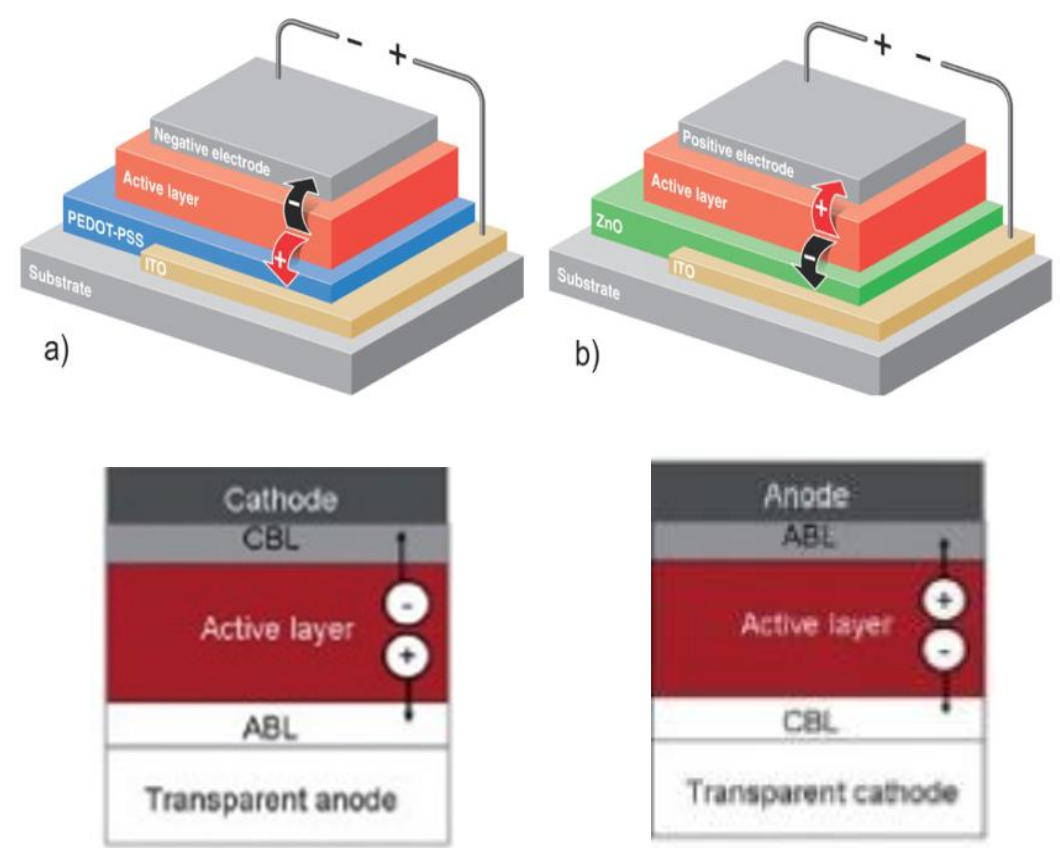

Figure 8. A Conventional (a) and inverted (b) solar cell [21]

The above Figure illustrates conventional and inverted organic solar cells. Although conventional and inverted OSCs are quite different in structure, their working principles are essentially the same. But examining the performance of the inverted structure in OSCs, it was found to protect the OSC from rapid degradation caused by some external factors.

Figure 9 shows the comparison of a conventional OSC and an inverted OSC in time domain. When this graph is examined, it will be seen that the sensitivity of the inverted OSC to this degradation parameter decreases. However, studies have shown that oxygen is still the fastest degrading parameter even for inverted OSCs [22]. 


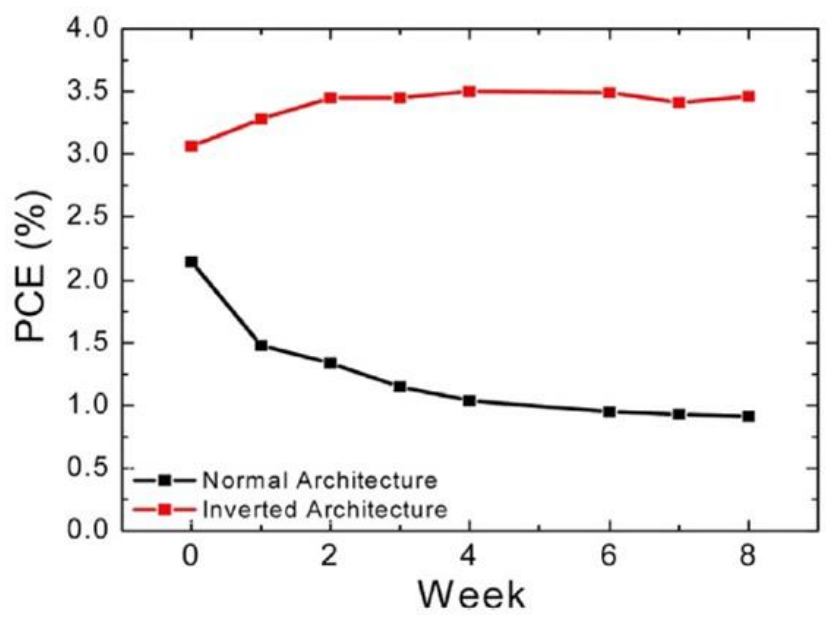

Figure 9. Comparison of Power Conversion Efficiencies of Conventional and Inverted OSCs [1].

The PEDOT:PSS component, which is the top layer of an inverted OSC, suffers significant degradation due to the phase change that oxygen causes [23].

According to Wang et al. they achieved a considerable improvement in CuPc/C60 solar cells, which they manufactured in a smaller molecular structure, by bringing it in a structure that is stable for 950 hours in the air environment [24]. They interpreted the reason to be that it takes longer for the moisture in the atmosphere to intrude the bottom layer of the OSC and diffuse into the PAL. In Figure 9, the Power Conversion Efficiencies (PCE) of OSCs with conventional (black) and inverted (red) architecture are plotted against time.

\section{Conclusion}

No need to mention again, OSCs should be stable, intrinsically and extrinsically. Furthermore, for the sake of flexibility, which modern solar cells really seem to need, ITO electrodes can be replaced with organic conducting materials, like PEDOT:PSS [25], or single-walled carbon nanotubes [26]. Finally, the organic semiconductors used in OSCs need to be chemically stable under solar irradiation [5, 27]. Stabilization of OSCs is structure dependent. In this section, we first briefly discuss a few ways of improving the intrinsic stability of OSCs, and then we discuss a few common ways of improving the extrinsic stability of conventional and inverted OSCs. Two general ways are to use metal oxides as the buffer layers, and silver as the top anodes. We further discuss two newly developed effective stabilization methods, with each only applicable to one type of structure. Titanium-aluminum can be used as the top cathode in conventional structures, whereas modified ITO can be used as the bottom cathode in inverted structures [1].

Degradation parameters have been found to have a significant impact on the operating stability of organic solar cells. In modern OSCs, these degradation parameters are tried to be minimized. Literature reports plenty of changes made to improve the operational stability of OSCs. In all these studies, the key is the development of a cathode-acceptor layer.

When the structure of an Inverted OSC is examined, it is obvious that a buffer layer is aimed to be formed by using Indium Tin Oxide polymer material as a lower layer, and metals, such as Ag, in the upper layer. With this change, the effect of moisture in the atmosphere on degradation of a conventional OSCs has been significantly reduced for in inverted OSCs. As a result, and the changes made in the design and in the layer structure of the organic solar cells will minimize the degradation and increase the operating stability of the system. Thus, the energy yield from solar cells will be increased.

\section{References}

[1] Cao, H.Q., et al., Recent progress in degradation and stabilization of organic solar cells. Journal of Power Sources, 2014. 264: p. 168-183.

[2] Yu, G., et al., Polymer Photovoltaic Cells - Enhanced Efficiencies Via a Network of Internal DonorAcceptor Heterojunctions. Science, 1995. 270(5243): p. 1789-1791.

[3] You, J.B., et al., A polymer tandem solar cell with $10.6 \%$ power conversion efficiency. Nature Communications, 2013. 4.

[4] Jorgensen, M., et al., Stability of Polymer Solar Cells. Advanced Materials, 2012. 24(5): p. 580-612. 
[5] Manceau, M., et al., Photochemical stability of pi-conjugated polymers for polymer solar cells: a rule of thumb. Journal of Materials Chemistry, 2011. 21(12): p. 4132-4141.

[6] Wang, F.Z., et al., Recent advances in planar heterojunction organic-inorganic hybrid perovskite solar cells. Acta Physica Sinica, 2015. 64(3).

[7] Bakulin, A.A., et al., The Role of Driving Energy and Delocalized States for Charge Separation in Organic Semiconductors. Science, 2012. 335(6074): p. 1340-1344.

[8] Group, T.R. and T.U.o.S. California. 2017 [cited 201723 May]; Available from: http://met.usc.edu/projects/solarcells.php.

[9] Schilinsky, P., et al., Simulation of light intensity dependent current characteristics of polymer solar cells. Journal of Applied Physics, 2004. 95(5): p. 2816-2819.

[10] Qi, B. and J. Wang, Fill factor in organic solar cells. Phys Chem Chem Phys, 2013. 15(23): p. 8972-82.

[11] Qi, B.Y. and J.Z. Wang, Open-circuit voltage in organic solar cells. Journal of Materials Chemistry, 2012. 22(46): p. 24315-24325.

[12] Scharber, M.C., et al., Design rules for donors in bulk-heterojunction solar cells - Towards $10 \%$ energyconversion efficiency. Advanced Materials, 2006. 18(6): p. 789-+.

[13] Wikipedia. 2017 [cited 201723 May]; Available from: https://en.wikipedia.org/wiki/PEDOT:PSS.

[14] Energy, S. 2017 [cited 201723 May]; Available from: http://solarmer.com/aboutus/.

[15] Song, Q.L., et al., Role of buffer in organic solar cells using C-60 as an acceptor. Applied Physics Letters, 2007. 90(7).

[16] Wahlstrom, E., et al., Electron transfer-induced dynamics of oxygen molecules on the TiO2(110) surface. Science, 2004. 303(5657): p. 511-513.

[17] Cuentas-Gallegos, A., et al., Electrochemical supercapacitors based on novel hybrid materials made of carbon nanotubes and polyoxometalates. Electrochemistry Communications, 2007. 9(8): p. 2088-2092.

[18] Vaillant, J., et al., Chemical synthesis of hybrid materials based on PAni and PEDOT with polyoxometalates for electrochemical supercapacitors. Progress in Solid State Chemistry, 2006. 34(24): p. 147-159.

[19] Krebs, F.C., T. Tromholt, and M. Jorgensen, Upscaling of polymer solar cell fabrication using full rollto-roll processing. Nanoscale, 2010. 2(6): p. 873-886.

[20] Xi, X., et al., A comparative study on the performances of small molecule organic solar cells based on CuPc/C-60 and CuPc/C-70. Solar Energy Materials and Solar Cells, 2010. 94(12): p. 2435-2441.

[21] Li, G., et al., Efficient inverted polymer solar cells. Applied Physics Letters, 2006. 88(25).

[22] Sondergaard, R., et al., Roll-to-roll fabrication of polymer solar cells. Materials Today, 2012. 15(1-2): p. 36-49.

[23] Norrman, K., et al., Degradation Patterns in Water and Oxygen of an Inverted Polymer Solar Cell. Journal of the American Chemical Society, 2010. 132(47): p. 16883-16892.

[24] Wang, M.L., et al., Small-molecular organic solar cells with C-60/Al composite anode. Organic Electronics, 2007. 8(4): p. 445-449.

[25] Zhou, Y.H., et al., A Universal Method to Produce Low-Work Function Electrodes for Organic Electronics. Science, 2012. 336(6079): p. 327-332.

[26] Rowell, M.W., et al., Organic solar cells with carbon nanotube network electrodes. Applied Physics Letters, 2006. 88(23).

[27] Lee, J.U., et al., Degradation and stability of polymer-based solar cells. Journal of Materials Chemistry, 2012. 22(46): p. 24265-24283.

\section{BIOGRAPHY OF AUTHORS}

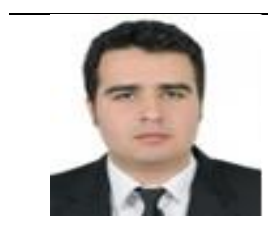

Efe Çetin YILMAZ was born in 1989. He received MSc degree from Mechanical Engineering the Ataturk University, Erzurum, Turkey, in 2014. Now, he is continuing from same University Ph.D. degree since in 2014. For five years, he has been working as a Lecturer in the Department of electronics and automation at Ataturk University. His areas of interest are control system design and programming, wear of biomaterials, corrosion and renewable energy. 


Muhammet Kaan YEŞiLYURT was born in 1979. After he received his Bsc in 2003, he worked for
many international companies, especially in oil and gas sector. Then he returned his academic career and
received his MSc at Ataturk University in 2015. He is working as an Instructor at Pasinler Vocational
School in Electric and Energy Department. He is now a PhD student at Ataturk University in Turkey.
IIhan Volkan ÖNER was born in 1979. He received MSc degree in Mechanical Engineering at Ataturk
University, Erzurum, Turkey, in 2009. Later, he received his Ph.D. degree also at Ataturk University in
2014. Since 2015, he has been working as an Assistant Professor in Department of Alternative Energy
Sources at Ataturk University. His areas of interest are renewable energy, internal combustion engines
and alternative fuels.
Gökhan ÖMEROĞLU was born in 1982. He received Bsc, MSc and Ph.D degrees at Ataturk University
in 2003, 2009, and 2012 respectively. He has started his career at Bayburt University as an Instructor.
After receiving his Ph.D he has been assigned the Assistant Professor position at the same university, and
shifted to Ataturk University the same year. Since then, he has been working as an assistant professor at
Ataturk University Engineering Faculty, Department of Mechanical Engineering, Thermodynamics
division.

Note: This study has been extended and revised after been presented in $3^{\text {rd }}$ International Conference on Engineering and Natural Science held on 3 - 7 May 2017 in Budapest, Hungary. It was previously published as Abstract in the Book of Abstracts of the conference. 